\title{
Correction to: Dietary obesity and glycemic excursions cause a parallel increase in STEAP4 and pro-inflammatory gene expression in murine PBMCs
}

\author{
Sigfrid Casmir Shayo ${ }^{1,2} \cdot$ Kazuma Ogiso $^{1} \cdot$ Shigeru Kawade $^{1} \cdot$ Hiroshi Hashiguchi ${ }^{1} \cdot$ Takahisa Deguchi $^{1}$. \\ Yoshihiko Nishio ${ }^{1}$
}

Published online: 17 October 2021

(C) The Japan Diabetes Society 2021

\section{Correction to: Diabetology International https://doi.org/10.1007/s13340-021-00542-1}

The original article was updated.

In the original publication of the article, figures D1 and D2 were missed in Fig. 7. The correct Fig. 7 is provided below.

The original article can be found online at https://doi.org/10.1007/ s13340-021-00542-1.

Yoshihiko Nishio

ynishio@m3.kufm.kagoshima-u.ac.jp

1 Department of Diabetes and Endocrine Medicine, Kagoshima University Graduate School of Medical and Dental Science, 8-35-1 Sakuragaoka,

Kagoshima 890-8520, Japan

2 Muhimbili University of Health and Allied Sciences, P.O.BOX 65001, Dar es Salaam, Tanzania 
A

A1

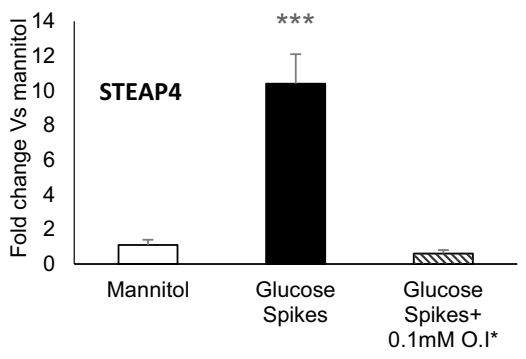

A3

B B1.
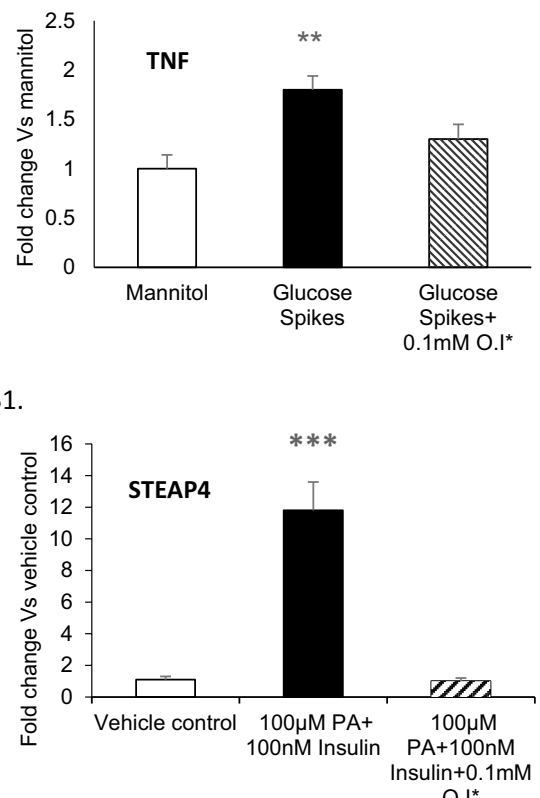

B3.

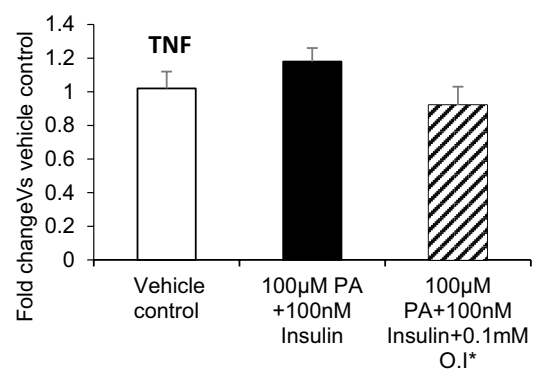

Fig.7 A The mRNA expression of (a1) STEAP4 (a2) CCL2 (a3) TNF and (a4) IL1B after 72 hours exposure of RAW 264.7 cells to either 8/16 hours repetitive cycles of mannitol osmolar fluctuations (control group) glucose fluctuations or $0.1 \mathrm{mM}$ of 4-octyl itaconate at every peak of glucose fluctuation (RT-qPCR) Data expressed as a fold change normalized to GAPDH Values are Means $\pm \operatorname{SEM}(n=5-5-5) * * p<0.01 * * * p<0.001$ against the mannitol osmolarity-control group O.I* stands for 4-octyl itaconate. B The mRNA expression of (b1) STEAP4 (b2) CCL2 (b3) TNF and (b4) IL1B after 24 hours exposure of RAW 264.7 cells to either a vehicle control (BSA) a combination of $100 \mu \mathrm{M}$ palmitic acid and $100 \mathrm{nM}$ Insulin or a combination of $100 \mu \mathrm{M}$ palmitic acid and $100 \mathrm{nM}$ Insulin and $0.1 \mathrm{mM}$ of 4-octyl itaconate (RTqPCR)Data expressed as a fold change normalized to GAPDH Values are Means $\pm \operatorname{SEM}(n=5-5-5) * * p<0.001$ against the vehicle-control group O.I* stands for 4-octyl itaconate. C The effect of (c1) PDTC an inhibitor of
A2

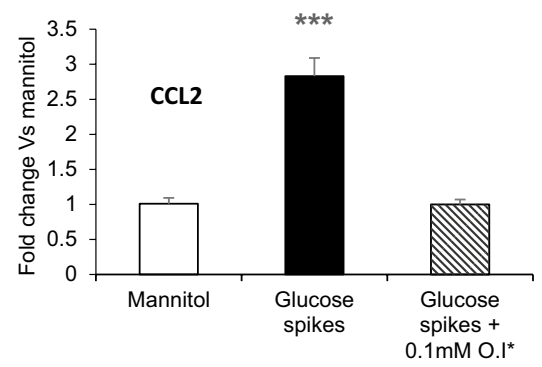

A4

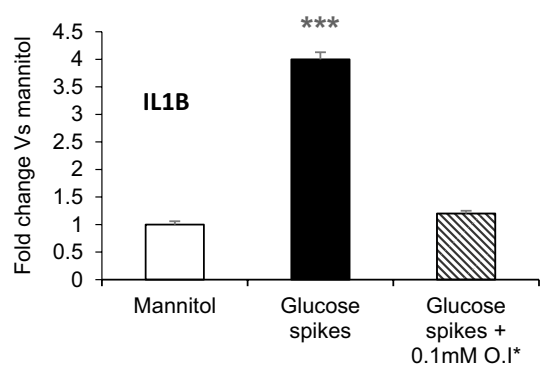

B2.

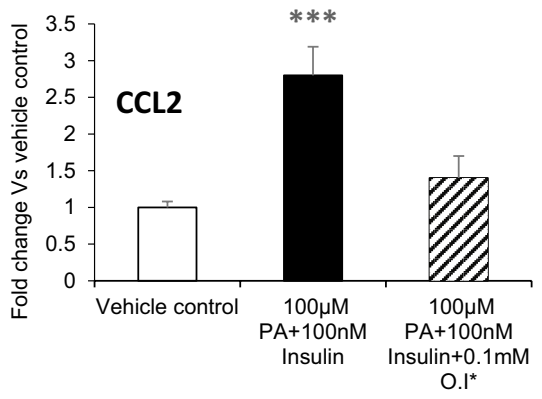

B4.

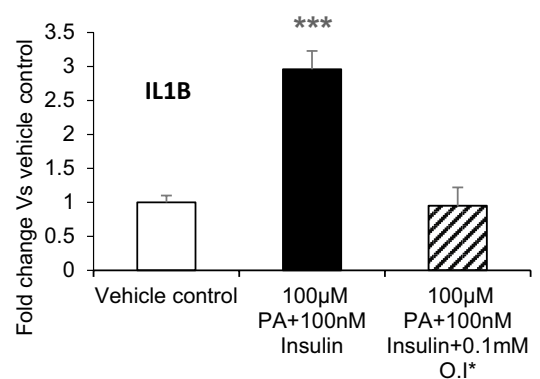

NF- $\mathrm{kB}$ activation on glucose spikes-induced STEAP4 mRNA expression in RAW 264.7 cells (c2) SB203580 a 38 MAPK inhibitor on glucose spikesinducedSTEAP4 mRNA expression in RAW 264.7 cells (c3) PDTC an inhibitor of NF- $\mathrm{KB}$ activation on persistent high-glucose induced STEAP4 mRNA expression in RAW 264.7 cells (c4) SB203580 a p38 MAPK inhibitor on persistent high-glucose induced STEAP4 mRNA expression in RAW 264.7 cells Dataexpressed as a fold change normalized to GAPDH Values are Means \pm SEM $(n=5-5-5) * * * p<0.001 * * p<0.01 * p<0.05$ NS means not statistically significant. D The effect of (d1) PDTC an inhibitor of NF- $\kappa \mathrm{B}$ activation on palmitic acid-high insulin-induced STEAP4 mRNA expression in RAW 264.7 cells (d2) SB203580 a p38 MAPK inhibitor on palmitic acid high insulin-induced STEAP4 mRNA expression in RAW 264.7 cells Data expressed as a fold change normalized to GAPDH Values are Means $\pm \operatorname{SEM}(n=5-5-5) * * * p<0.001 * * p<0.01$ 
C

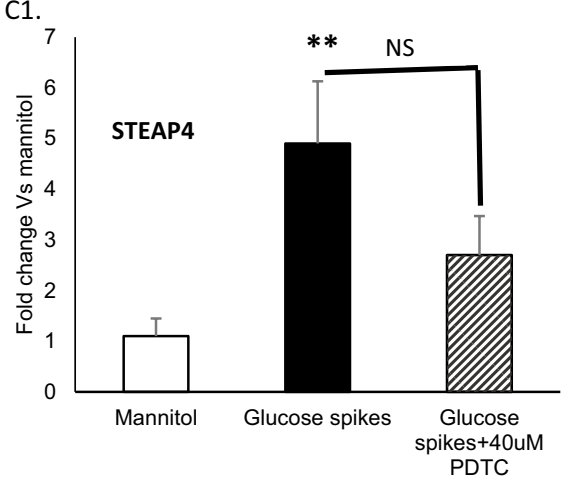

C3.

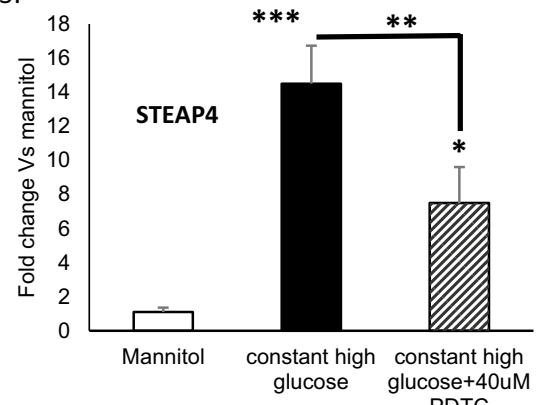

D D1.

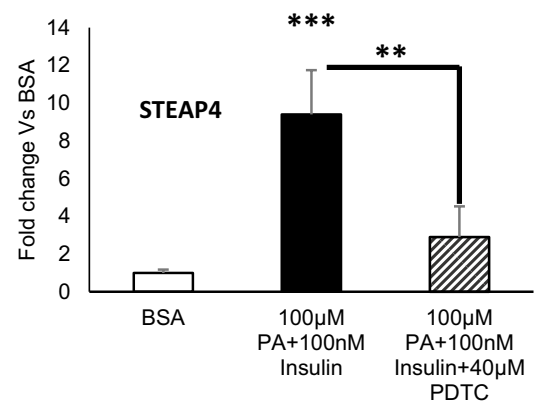

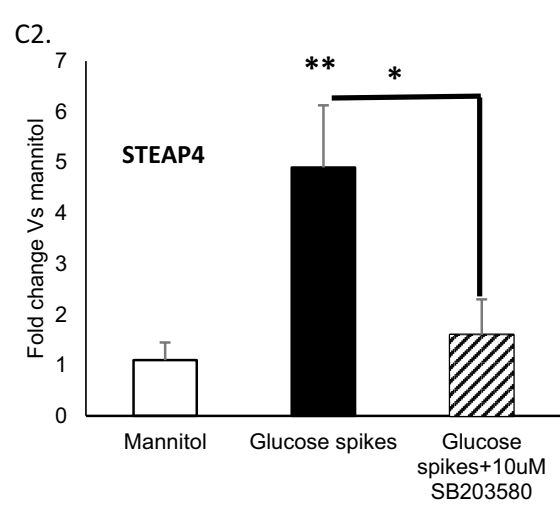

C4.

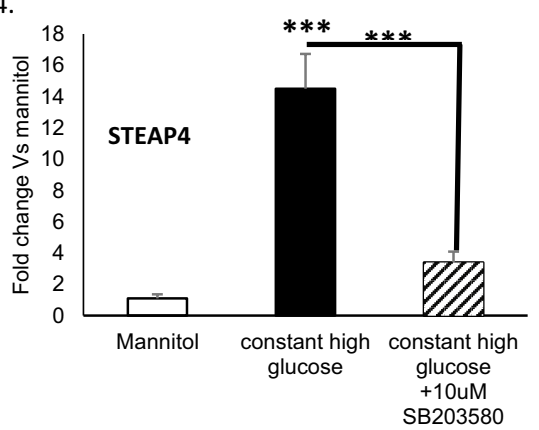

D2.

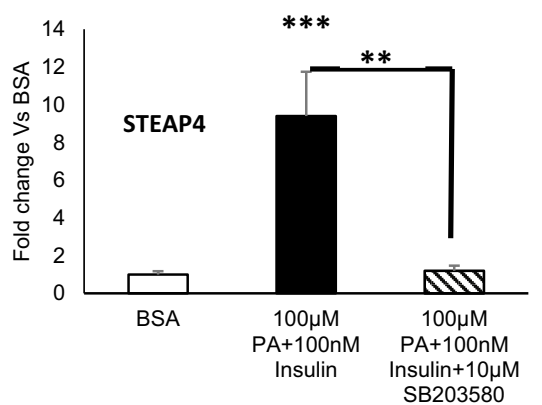

Fig. 7 (continued)

Publisher's Note Springer Nature remains neutral with regard to jurisdictional claims in published maps and institutional affiliations. 